\title{
Rent-Seeking Contests with Incomplete Information
}

\author{
Mark Fey* \\ Department of Political Science \\ University of Rochester
}

September, 2007

\begin{abstract}
We consider rent-seeking contests with two players that each have private information about their own cost of effort. We consider both discrete and continuous distributions of costs and give results for each case, focusing on existence of equilibria.

JEL Classification: D72; C72

Keywords: rent-seeking, contests, conflict, private information, equilibrium existence
\end{abstract}

${ }^{*}$ Harkness Hall, University of Rochester, Rochester, NY, 14618. Office: 585-275-5810. Fax: 585-271-1616. Email: markfey@mail.rochester.edu. 


\section{Introduction}

A rent-seeking contest is a situation in which players expend costly effort to gain a reward. Many conflict situations can be described by rent-seeking contests, including political campaigns, patent races, war fighting, lobbying efforts, labor market competition, legal battles, and professional sports. The reward in a rent-seeking contest may be indivisible, such as electoral office or a patent right, or it may be divisible, such as market share or vote share. In the former case, expending more effort increases the probability that a player will win the prize. In the latter case, expending more effort increases the share of the prize.

An important vehicle for investigating the logic of rent-seeking contests is the model of Tullock (1980). This work has spawned a large literature, some of which is surveyed in Lockard and Tullock (2001) and Corchón (2007). In this paper, we contribute to this literature by developing a model of rentseeking contests in which players have incomplete information about the cost of effort to other players. That is, players are uncertain about the value of others for expending effort. ${ }^{1}$

We consider rent-seeking contests with two players that each have private information about their own cost of effort. We model such a contest as Bayesian game in which each player's cost is drawn from a distribution of possible costs. We consider both discrete and continuous distributions of costs and give results for each case. We focus on existence of equilibria, which, in the continuous case, requires the use of a functional analysis fixed point argument.

The focus on rent-seeking contests with two-sided incomplete information and continuous cost distributions is what sets this paper apart from other papers that consider asymmetric information in contests. For exam-

\footnotetext{
${ }^{1}$ It is important to distinguish this informational type of uncertainty from the uncertainty due to risk aversion that has been examined in the literature (Hillman and Katz, 1984; Van Long and Vousden, 1987).
} 
ple, Harstad (1995) looks at a model with asymmetrically informed agents, but in the context of a winner-take-all game. Hurley and Shogren (1998b) and Schoonbeek and Winkel (2006) both consider models with one-sided incomplete information, while Hurley and Shogren (1998a) and Malueg and Yates (2004) present models with two-sided incomplete information in which both players' valuations are either high or low. Additionally, recent work on existence of equilibria in rent-seeking contests includes Cornes and Hartley (2005) and Malueg and Yates (2006), but these papers assume complete information. Bernardo et al. (2000) embed a rent-seeking contest with onesided incomplete information in a moral hazard model of litigation. Finally, Wärneryd (2003) gives a Bayesian model of rent-seeking contests in which there is asymmetric information about the common value of the prize. Although we deal with the private value case of individual cost of effort, some of the techniques of analysis are similar.

The paper is organized in the following fashion. The next section contains the definitions and notation of the model, as well as a summary of the results for the complete information setting. In section 3 , we examine a simple example of incomplete information in which each player has only two possible costs of effort. We explicitly solve for the equilibrium and provide some comparative statics. In section 4, we consider the continuous case, with a uniform distribution of possible costs. The main result is an existence proof. We also graphically present a numerical estimate of the equilibrium and provide some comparative statics and properties for this case. Finally, section 5 concludes.

\section{Notation and Definitions}

We model a rent-seeking contest as a game between two players who must choose a level of costly effort to obtain a share of a prize. Each player chooses a level of effort $e_{i} \geq 0$ for $i=1,2$. The effort level of both players is denoted 
$e=\left(e_{1}, e_{2}\right)$. The effort levels chosen by the players determine a portion $f_{i}(e) \in[0,1]$ for player $i$. We interpret a "portion" in one of two ways either as a fraction of a divisible good or as the probability of receiving a nondivisible good. By the expected utility theorem, these interpretations are mathematically equivalent if both players are risk neutral. ${ }^{2}$ We assume that the benefit a player receives is linear in the player's portion and the cost a player pays is linear in effort. That is, there are constants $\alpha_{i}, \beta_{i}, \gamma_{i}$, and $\delta_{i}$ with $\beta_{i}>0$ and $\delta_{i}>0$, such that player $i$ 's expected utility is

$$
u_{i}(e)=\alpha_{i}+\beta_{i} f_{i}(e)-\left(\gamma_{i}+\delta e_{i}\right)
$$

Without loss of generality, we can take $\alpha_{i}-\gamma_{i}=0$. Likewise, we can divide by $\beta_{i}$, which gives

$$
u_{i}(e)=f_{i}(e)-\frac{\delta_{i}}{\beta_{i}} e_{i} .
$$

To operationalize the analysis, we specify the following functional form for $f_{i}(e)$ :

$$
f_{i}(e)= \begin{cases}e_{i} / \sum_{j} e_{j} & \text { if } \sum_{j} e_{j}>0 \\ 1 / 2 & \text { otherwise. }\end{cases}
$$

This functional form is common in the literature (Tullock, 1980; Nitzan, 1991; Szidarovsky and Okuguchi, 1997) and is a simple form of the contest success functions axiomatized by Skaperdas (1996) and Clark and Riis (1998). ${ }^{3}$ Letting $c_{i}=\delta_{i} / \beta_{i}$, the expected utility for player $i$ is

$$
u_{i}(e)=\frac{e_{i}}{\sum_{j} e_{j}}-e_{i} c_{i}
$$

unless $e_{i}=0$, in which case $u_{i}(e)=0$. By defining $c_{i}$ in this way, we include the case in which the individuals' valuations of the prize (the $\beta_{i}$ term) differ

\footnotetext{
${ }^{2}$ This is not true if the player are risk averse (Hillman and Katz, 1984; Van Long and Vousden, 1987).

${ }^{3}$ In fact, our results do not depend on the value of $f_{i}(e)$ at $e=(0,0)$.
} 
(Hillman and Riley, 1989).

If both players have complete information about all aspects of the game, the equilibrium of this game is easily found (and well known). If the players have the same cost of effort, $c$, then the first order condition for player $i$ is

$$
\frac{e_{-i}}{\left(\sum_{j} e_{j}\right)^{2}}=c
$$

As this must hold for both players, it clear that, in equilibrium, $e_{1}=e_{2}{ }^{4}$ Substituting this back into equation (1) gives the solution $e_{1}=e_{2}=1 /(4 c)$ in equilibrium. As both players would be strictly better off by choosing $e_{1}=e_{2}=0$, this equilibrium solution is inefficent, again, a standard result.

Building on this complete information setup, in this paper we model a contest as a game of incomplete information in which players are uncertain about the other's cost of effort. ${ }^{5}$ In the sections that follow, we consider both the discrete and continuous cases. We introduce some general notation here and give precise details in the later sections.

We suppose that each player's cost is drawn independently from a distribution $G(c)$ before the game is played. $G(c)$ may be discrete or continuous and we denote the support of $G$ by $C \subseteq \mathbb{R}_{+}$. Each player's own cost is private information and thus $G$ represents the players' common prior of the type of the other, where we identify the type of a player with their cost. A (pure) strategy for player $i$ is a function $\sigma_{i}: C \rightarrow \mathbb{R}_{+}$. In other words, a player $i$ with cost $c_{i}$ chooses an effort level $e_{i}=\sigma_{i}\left(c_{i}\right)$.

The equilibrium concept we use for this simultaneous Bayesian game is Bayesian-Nash equilibrium. Specifically, a strategy profile $\left(\sigma_{1}^{*}, \sigma_{2}^{*}\right)$ is a Bayesian-Nash equilibrium if, for every $c_{1} \in C$,

$$
\int_{C} u_{1}\left(\sigma_{1}^{*}\left(c_{1}\right), \sigma_{2}^{*}(c)\right) d G(c) \geq \int_{C} u_{1}\left(\sigma_{1}^{\prime}\left(c_{1}\right), \sigma_{2}^{*}(c)\right) d G(c)
$$

\footnotetext{
${ }^{4}$ It is easy to check that $e_{i}>0$ holds for both players in equilibrium.

${ }^{5}$ See Gradstein (1995) for a model with variable effort effectiveness instead of variable costs.
} 
for every $\sigma_{1}^{\prime}$, and similarly for player 2 . We restrict our attention to symmetric equilibria. These are equilibria in which players of the same type take the same action. In our setting, a symmetric Bayesian-Nash equilibrium satisfies $\sigma_{1}^{*}=\sigma_{2}^{*}=\sigma^{*}$.

\section{Results: Discrete Case}

In this section, we analyze the simplest possible formulation of this game of incomplete information; namely, the discrete case with only two types for each player. This simple formulation of uncertainty has been previously examined by Hurley and Shogren (1998a) and Malueg and Yates (2004). In particular, we assume that $C=\left\{c_{L}, c_{H}\right\}$, with $c_{H}>c_{L}>0$, and that each player $i$ (independently) draws cost $c_{i}=c_{L}$ with probability $1 / 2$ and draws $\operatorname{cost} c_{i}=c_{H}$ with probability $1 / 2$. Thus, a (symmetric) strategy is a function $\sigma: C \rightarrow \mathbb{R}_{+}$. As the domain of this function has only two elements, it is sufficient to consider two values, $e_{L}$ and $e_{H}$, defined by $e_{L}=\sigma\left(c_{L}\right)$ and $e_{H}=\sigma\left(c_{H}\right)$.

The first order condition is given by

$$
\left(\frac{1}{2}\right) \frac{e_{H}}{\left(e_{i}+e_{H}\right)^{2}}+\left(\frac{1}{2}\right) \frac{e_{L}}{\left(e_{i}+e_{L}\right)^{2}}=c_{i} .
$$

When $c_{i}=c_{L}, e_{i}=e_{L}$ must satisfy this condition. This gives

$$
\frac{e_{H}}{\left(e_{L}+e_{H}\right)^{2}}+\frac{1}{4 e_{L}}=2 c_{L}
$$

Likewise, when $c_{i}=c_{H}, e_{i}=e_{H}$ must satisfy the first order condition, ${ }^{6}$ so

$$
\frac{1}{4 e_{H}}+\frac{e_{L}}{\left(e_{L}+e_{H}\right)^{2}}=2 c_{H} .
$$

\footnotetext{
${ }^{6}$ It is easy to show that $e_{L}$ and $e_{H}$ must both be positive.
} 
Manipulating equations 3 and 4 gives

$$
4 e_{L} e_{H}+\left(e_{L}+e_{H}\right)^{2}=2 c_{L}\left(4 e_{L}\right)\left(e_{L}+e_{H}\right)^{2}
$$

and

$$
\left(e_{L}+e_{H}\right)^{2}+4 e_{H} e_{L}=2 c_{H}\left(4 e_{H}\right)\left(e_{L}+e_{H}\right)^{2} .
$$

From equations (5) and (6), it is clear that $c_{L} e_{L}=c_{H} e_{H}$ must hold. Equivalently, we must have

$$
\frac{e_{H}}{e_{L}}=\frac{c_{L}}{c_{H}}
$$

In other words, the ratio of the equilibrium effort levels must be the inverse of the ratio of the costs. ${ }^{7}$ Plugging $e_{H}=e_{L}\left(c_{L} / c_{H}\right)$ into equation (5) yields

$$
e_{L}=\frac{4 \frac{c_{L}}{c_{H}}+\left(1+\frac{c_{L}}{c_{H}}\right)^{2}}{8 c_{L}\left(1+\frac{c_{L}}{c_{H}}\right)^{2}}
$$

and thus by equation (7),

$$
e_{H}=\frac{4 \frac{c_{L}}{c_{H}}+\left(1+\frac{c_{L}}{c_{H}}\right)^{2}}{8 c_{H}\left(1+\frac{c_{L}}{c_{H}}\right)^{2}} .
$$

To summarize, in this simple discrete case, a unique (symmetric) equilibrium exists.

We now turn to analyzing the comparative statics of this case. It is straightforward but tedious to show that $\partial e_{L} / \partial c_{L}<0$. Thus, holding the high cost constant, increasing the low cost decreases the effort level of a low type. Perhaps a more interesting comparative static is the case in which both costs change in such a way that $\frac{c_{L}}{c_{H}}$ is held constant. Then it is immediate from equations (8) and (9) that the effort levels of both types of player change by an inverse amount. Thus, when both costs are doubled, for example, the effort levels of both types of player decrease by half.

\footnotetext{
${ }^{7}$ See Malueg and Yates (2004) for a similar result.
} 
Finally, consider what happens to the equilibrium as $c_{L}$ converges to $c_{H}$. From equation (7), it is clear that $e_{L}$ goes to $e_{H}$ as $c_{L}$ goes to $c_{H}$. Moreover, if $c_{L}$ and $c_{H}$ converge to some value $c$, then $e_{L}$ and $e_{H}$ must converge to $1 / 4 c$, which is the solution to the complete information version of the model.

\section{Results: Continuous Case}

In this section we prove existence and numerically analyze the equilibrium for the case of a continuous distribution of costs. By considering the case in which each player has a continuum of possible types, we allow for a much richer and more general specification of uncertainty than previous models which consider only two possible types of each player, such as Hurley and Shogren (1998a) and Malueg and Yates (2004).

For simplicity, we suppose that each player's $\operatorname{cost} c_{i}$ is drawn independently from a uniform distribution on an interval of length one, that is $G(c) \sim U[\underline{\mathrm{c}}, \underline{\mathrm{c}}+1]$, with $\underline{\mathrm{c}}>0$ and $C=[\underline{\mathrm{c}}, \underline{\mathrm{c}}+1]$. With this assumption, the expected utility for player $i$ with $\operatorname{cost} c_{i}$, when the opponent plays strategy $\sigma$, is given by

$$
u_{i}\left(e_{i}, \sigma ; c_{i}\right)=-e_{i} c_{i}+\int_{C} \frac{e_{i}}{e_{i}+\sigma(t)} d t .
$$

Because player $i$ can guarantee a payoff of at least zero by setting $e_{i}=0$, the optimal choice of $e_{i}$ must satisfy $u_{i}\left(e_{i}, \sigma ; c_{i}\right) \geq 0$ for all $c_{i} \in C$. As the integral in equation (10) is bounded by 1 , this implies that the optimal choice of $e_{i}$ must satisfy $0 \leq e_{i} \leq 1 / c_{i}$. On the interval $C=[\underline{\mathrm{c}}, \underline{\mathrm{c}}+1]$, then, an optimal choice of $e_{i}$ must satisfy $0 \leq e_{i} \leq 1 / \underline{\text {. }}$. We denote this upper bound by $E=1 / \underline{c}$.

A player with cost $c_{i}$ faces an optimization problem of maximizing equation (10) subject to $e_{i} \geq 0$. The Kuhn-Tucker conditions for a given value of 
$c_{i}$ are the following:

$$
\begin{array}{ll}
-c_{i}+\int_{C} \frac{\sigma(t)}{\left(e_{i}+\sigma(t)\right)^{2}} d t=0 & \text { for } e_{i}>0, \text { and } \\
-c_{i}+\int_{C} \frac{\sigma(t)}{\left(e_{i}+\sigma(t)\right)^{2}} d t \leq 0 & \text { for } e_{i}=0 .
\end{array}
$$

Examining the integral in equation (11), it is clear that it is strictly decreasing in $e_{i}$ and goes to zero as $e_{i}$ gets large. Thus, it follows that this first order condition is satisfied by exactly one value of $e_{i}$. A best response for player $i$ to $\sigma$, which we denote $b_{\sigma}(c)$ or $b(c)$, is precisely the value of $e_{i}$ that satisfies the first order condition with cost $c$. Thus, the best response function $b_{\sigma}(c)$ is well-defined, nonnegative, and decreasing in $c .^{8}$ In addition, it is an easy consequence of the implicit function theorem that the best response function is continuous.

We now present the main result of this section - that a equilibrium exists in our model.

Theorem 1 If $G\left(c_{i}\right) \sim U[\underline{c}, \underline{c}+1]$, with $\underline{c}>0$, for $i=1,2$, then a symmetric pure strategy Bayesian-Nash equilibrium exists.

The proof of this theorem is contained in the appendix. As is standard, the method of proof of existence is to find a fixed point of the best response mapping. However, this mapping is defined on a subset of a space of functions which, like most such sets, is not compact (under the standard sup norm). Therefore we use an alternative approach. We have already established that the best response functions are monotone and continuous. In the appendix, we show that the set of best response functions is equicontinuous and that the operator that maps the opponent's strategy into a best response function is continuous. This allows us to establish existence using Schauder's Fixed Point Theorem. This approach is more flexible than a contraction mapping

\footnotetext{
${ }^{8} \mathrm{In}$ fact, it is clear that $b(c)$ is strictly decreasing for values of $c$ with $b(c)>0$.
} 


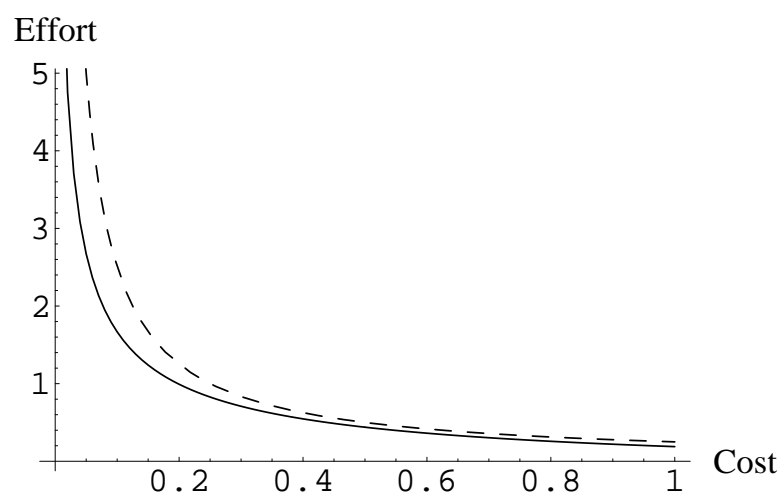

Figure 1. Numerical solution and comparison to complete information

argument (which only requires completeness), but it does not insure uniqueness of the fixed point. We conjecture that the equilibrium to the model is, in fact, unique, but leave a proof for future work.

Theorem 1 shows that an equilibrium exists. To find a closed-form solution for it, we must solve the integral equation given in equation (11) for all $c \in C$. One approach to accomplish this is by working with the inverse of the solution, which transforms the integral equation from nonlinear into linear form, but such an approach appears to be intractable in this case. On the other hand, it is possible to numerically calculate a solution by a standard iterative method. For the case of $\underline{\mathrm{c}}=.01$, the numerical solution is shown in Figure 1. In the figure, the horizontal axis is the cost value drawn by a player and the vertical axis is the equilibrium effort expended. The numerical solution is given by the solid line in the figure. This solution was calculated numerically over a grid of 100 elements and thus the figure is a good approximation of the true equilibrium strategy.

Figure 1 also illustrates how the equilibrium effort level under incomplete information compares to the effort level of the complete information symmetric game from section 2. Under complete information, the equilibrium effort level is $e_{i}=1 /(4 c)$, which is indicated by the dashed line in Figure 1 . It is interesting to observe that the two functions are qualitatively similar, but 
the incomplete information strategy always calls for slightly less effort than the complete information version. ${ }^{9}$ This result echoes an earlier finding by Hurley and Shogren (1998a) that, in a model in which each player could have either a high or low valuation of the prize, asymmetric information makes effort more risky, which tends to decrease effort levels. Figure 1 illustrates that this effect carries over to a model in which both players have a continuum of possible types.

Finally, inspection of Figure 1 shows that both players always exert positive effort in equilibrium. In fact, this is true for any symmetric equilibrium of this game. To see this, suppose that there is a symmetric equilibrium in which a non-zero interval of costs gives zero effort. Let the probability that such a cost is realized be $p_{0}>0$. If this occurs, the other player can gain the prize with certainty by exerting an arbitrarily small amount of effort. In particular, a level of effort that satisfies $0<e_{i}<p_{0} / c_{i}$ yields a gain in expected utility. With this choice of effort at cost $c_{i}$, a player does strictly better than with $e_{i}=0$. Thus, there can be no interval of costs for which zero effort is optimal in a symmetric equilibrium. Moreover, a similar argument shows that this result continues to hold in any symmetric equilibrium of a game with $n>2$ players. ${ }^{10}$ This conclusion differs from work on entry in complete information rent-seeking contests in which some players choose not to participate by choosing zero effort (Gradstein, 1995; Higgins et al., 1985). ${ }^{11}$ However, such situations under complete information can occur only in "asymmetric" contests in which players have different valuations. Our finding that players always exert positive effort under incomplete information applies to symmetric equilibria of a symmetric model (i.e., all players have an identical cost distribution). Thus, even though players can

\footnotetext{
${ }^{9}$ Of course, the dashed line is not a strategy in the same way that the solid line is, but rather describes equilibrium effort as the cost of effort in the complete information game is varied.

${ }^{10}$ The only modification required in the above argument is that the level of effort must satisfy $0<e_{i}<p_{0}^{n-1} / c_{i}$ in a game with $n$ players.

${ }^{11}$ But see Wärneryd (2006) for a similar result in a model with asymmetric information.
} 
have different realized costs in our model, they are identical ex ante. These differences in the symmetry of the models suggest that caution is warranted in making comparisons between them.

\section{Conclusion}

In this paper, we have examined rent-seeking contests under various forms of incomplete information. The analysis of these models has been complex in places, but we have gained some insight into the role that information plays in this class of games. Our main result is a proof of existence of equilibrium when costs are drawn from a continuous distribution.

Much work remains to be done in this vein, particulary for the continuous case. First, the model can be made more general by allowing for more general distributions of costs and more than two players. Fortunately, it appears that in both cases, the line of proof given in the appendix should go through with only minor modifications. However, numerical calculation of the result would be more difficult. A second line of work involves generalizing the contest success function $f_{i}(e)$. It is likely this will be a more difficult task to accomplish. Another aspect of the problem that remains open is an examination of efficiency in the continuous case. Direct comparison with the complete information equilibrium demonstrates that inefficiency is still prevalent, but more work in this area remains to be done. Finally, we have restricted our attention to symmetric equilibria in this paper. Future work that considers asymmetric equilibria in this model and/or asymmetric models of rent-seeking contests may be illuminating and ultimately more realistic. 


\section{Appendix: Proof of Existence}

Theorem If $G\left(c_{i}\right) \sim U[\underline{c}, \underline{c}+1]$, with $\underline{c}>0$, for $i=1,2$, then a symmetric Bayesian-Nash equilibrium exists.

Proof: Let $C[a, b]$ be the space of all real continuous functions on the interval $[a, b]$. Equipped with the sup norm, $\|f(x)\|=\sup _{x \in[a, b]}|f(x)|$, this space is a Banach space. We are interested in the set of nonnegative continuous functions on the interval $C=[\underline{\mathrm{c}}, \underline{\mathrm{c}}+1]$ bounded by $E=1 / \underline{\mathrm{c}}$. Formally, define

$$
C_{E}[\underline{\mathrm{c}}, \underline{\mathrm{c}}+1]=\{f \in C[\underline{\mathrm{c}}, \underline{\mathrm{c}}+1] \mid\|f\| \leq E \text { and } f(x) \geq 0, x \in[\underline{\mathrm{c}}, \underline{\mathrm{c}}+1]\} .
$$

Clearly, this is a closed, convex subset of $C[\underline{\mathrm{c}}, \underline{\mathrm{c}}+1] .{ }^{12}$

For a given function $\sigma \in C_{E}$, let the best response function $b_{\sigma}(c)$ be as defined above. As $b_{\sigma}(c)$ is bounded by $E$ and is continuous, $b_{\sigma}(c) \in C_{E}$. Therefore we can define $T: C_{E} \rightarrow C_{E}$ to be the mapping $\sigma \mapsto b_{\sigma}$. As the best response function is unique, this mapping is a function. An equilibrium is a fixed point of this function, $\sigma^{*}=T \sigma^{*}$.

Because $C_{E}$ is not compact with the sup norm, we rely on a fixed point theorem for compact operators. An operator $S: X \rightarrow Y, X$ and $Y$ Banach spaces, is compact if it is continuous and maps bounded sets into relatively compact sets. ${ }^{13}$ We can now state the Schauder Fixed Point Theorem, a useful discussion of which is found in Zeidler (1986).

Theorem (Schauder) Let $X$ be a nonempty, closed, bounded, convex subset of a Banach space and suppose $S: X \rightarrow X$ is a compact operator. Then $S$ has a fixed point.

In order to establish that $T$ is a compact operator, the characterization of relative compactness in the space of continuous functions given by the Arzelà-

\footnotetext{
${ }^{12}$ For simplicity, in what follows we exclude from this set the constant zero function. Excluding this element would mean this set is not closed. This issue can be resolved by considering a sequence of subsets of $C_{E}$ requiring $\|f\| \geq \varepsilon$, with $\varepsilon \rightarrow 0$.

${ }^{13} \mathrm{~A}$ set is relatively compact if its closure is compact.
} 
Ascoli Theorem is very useful. This theorem uses the following two definitions. A set of (real-valued) functions $\mathcal{F}$ is equicontinuous on the interval $C$ if for every $\varepsilon>0$ there is a $\delta>0$ such that $|x-y|<\delta$ implies $|f(x)-f(y)|<\varepsilon$ for all $f \in \mathcal{F}$ and all $x, y \in C$. A set of (real-valued) functions $\mathcal{F}$ is uniformly bounded on the interval $C$ if there is a value $M<\infty$ such that $|f(x)| \leq M$ for all $f \in \mathcal{F}$ and all $x \in C$.

Theorem (Arzelà-Ascoli) A set of functions in $C[a, b]$, with the sup norm, is relatively compact if and only if it is uniformly bounded and equicontinuous on $[a, b]$.

Clearly $C_{E}$ is uniformly bounded, so to establish our result we must show that $T$ is continuous and has equicontinuous range.

We first show that the range of $T$ is a set of equicontinuous functions. From the first order condition, we have

$$
\begin{aligned}
c_{1}-c_{2}= & \int_{C} \frac{\sigma(t)}{\left(b\left(c_{1}\right)+\sigma(t)\right)^{2}} d t-\int_{C} \frac{\sigma(t)}{\left(b\left(c_{2}\right)+\sigma(t)\right)^{2}} d t \\
= & \int_{C} \frac{\sigma(t)}{\left(b\left(c_{1}\right)+\sigma(t)\right)^{2}}-\frac{\sigma(t)}{\left(b\left(c_{2}\right)+\sigma(t)\right)^{2}} d t \\
= & \int_{C} \sigma(t) \frac{\left(b\left(c_{2}\right)+\sigma(t)\right)^{2}-\left(b\left(c_{1}\right)+\sigma(t)\right)^{2}}{\left(b\left(c_{1}\right)+\sigma(t)\right)^{2}\left(b\left(c_{2}\right)+\sigma(t)\right)^{2}} d t \\
= & \int_{C} \sigma(t) \frac{b^{2}\left(c_{2}\right)-b^{2}\left(c_{1}\right)+2 \sigma(t)\left(b\left(c_{2}\right)-b\left(c_{1}\right)\right)}{\left(b\left(c_{1}\right)+\sigma(t)\right)^{2}\left(b\left(c_{2}\right)+\sigma(t)\right)^{2}} d t \\
= & {\left[b\left(c_{2}\right)-b\left(c_{1}\right)\right] \int_{C} \sigma(t) \frac{b\left(c_{2}\right)+b\left(c_{1}\right)+2 \sigma(t)}{\left(b\left(c_{1}\right)+\sigma(t)\right)^{2}\left(b\left(c_{2}\right)+\sigma(t)\right)^{2}} d t } \\
= & {\left[b\left(c_{2}\right)-b\left(c_{1}\right)\right] \int_{C} \sigma(t) \frac{b\left(c_{1}\right)+\sigma(t)+b\left(c_{2}\right)+\sigma(t)}{\left(b\left(c_{1}\right)+\sigma(t)\right)^{2}\left(b\left(c_{2}\right)+\sigma(t)\right)^{2}} d t } \\
= & {\left[b\left(c_{2}\right)-b\left(c_{1}\right)\right]\left[\int_{C} \frac{\sigma(t)}{\left(b\left(c_{1}\right)+\sigma(t)\right)\left(b\left(c_{2}\right)+\sigma(t)\right)^{2}}\right.} \\
& \left.\quad+\frac{\sigma(t)}{\left(b\left(c_{1}\right)+\sigma(t)\right)^{2}\left(b\left(c_{2}\right)+\sigma(t)\right)} d t\right] .
\end{aligned}
$$

The two integrals on this last line are both positive. Using the first order 
condition and the fact that $b(c)$ and $\sigma(t)$ are both bounded by $E$, we have

$$
\int_{C} \frac{\sigma(t)}{\left(b\left(c_{1}\right)+\sigma(t)\right)\left(b\left(c_{2}\right)+\sigma(t)\right)^{2}} d t \geq \int_{C} \frac{\sigma(t)}{2 E\left(b\left(c_{2}\right)+\sigma(t)\right)^{2}} d t=\frac{c_{2}}{2 E}
$$

A similar argument shows that the second integral in the expression above is bounded below by $c_{1} / 2 E$. Taking absolute values, we have

$$
\left|c_{1}-c_{2}\right| \geq\left|b\left(c_{2}\right)-b\left(c_{1}\right)\right|\left(\frac{c_{2}}{2 E}+\frac{c_{1}}{2 E}\right)>\left|b\left(c_{2}\right)-b\left(c_{1}\right)\right|\left(\frac{\underline{\mathrm{c}}}{E}\right)
$$

Now, fix $\varepsilon>0$ and let $\delta=\varepsilon(\underline{\mathrm{c}} / E)$. Then $\left|c_{1}-c_{2}\right|<\delta$ implies $\mid b\left(c_{2}\right)-$ $b\left(c_{1}\right) \mid(\underline{\mathrm{c}} / E)<\varepsilon(\underline{\mathrm{c}} / E)$ or $\left|b\left(c_{2}\right)-b\left(c_{1}\right)\right|<\varepsilon$. As this holds for all best response functions and all $c_{1}, c_{2} \in C$, this establishes equicontinuity.

We now show that the operator $T$ is continuous. In other words, if $\sigma_{n} \rightarrow \sigma$ and $b_{\sigma_{n}}=T \sigma_{n}$ and $b_{\sigma}=T \sigma$, then $b_{\sigma_{n}} \rightarrow b_{\sigma}$. Convergence in a function space with the sup norm is equivalent to uniform convergence, so we must show $b_{\sigma_{n}}$ converges uniformly to $b_{\sigma}$. So take a sequence $\sigma_{n} \rightarrow \sigma$ and a point $c \in C$. The first order condition yields

$$
\int_{C} \frac{\sigma_{n}(t)}{\left(b_{\sigma_{n}}(c)+\sigma_{n}(t)\right)^{2}} d t=c=\int_{C} \frac{\sigma(t)}{\left(b_{\sigma}(c)+\sigma(t)\right)^{2}} d t
$$

And thus

$$
\begin{gathered}
\int_{C} \frac{\sigma_{n}(t)}{\left(b_{\sigma_{n}}(c)+\sigma_{n}(t)\right)^{2}}-\frac{\sigma(t)}{\left(b_{\sigma}(c)+\sigma(t)\right)^{2}} d t=0 \\
\int_{C} \frac{\sigma_{n}(t)\left(b_{\sigma}^{2}(c)+2 b_{\sigma}(c) \sigma(t)+\sigma^{2}(t)\right)-\sigma(t)\left(b_{\sigma_{n}}^{2}(c)+2 b_{\sigma_{n}}(c) \sigma_{n}(t)+\sigma_{n}^{2}(t)\right)}{\left(b_{\sigma_{n}}(c)+\sigma_{n}(t)\right)^{2}\left(b_{\sigma}(c)+\sigma(t)\right)^{2}} d t=0 \\
\int_{C} \frac{\sigma_{n}(t) b_{\sigma}^{2}(c)-\sigma(t) b_{\sigma_{n}}^{2}(c)+2 \sigma(t) \sigma_{n}(t)\left(b_{\sigma}(c)-b_{\sigma_{n}}(c)\right)+\sigma(t) \sigma_{n}(t)\left(\sigma(t)-\sigma_{n}(t)\right)}{\left(b_{\sigma_{n}}(c)+\sigma_{n}(t)\right)^{2}\left(b_{\sigma}(c)+\sigma(t)\right)^{2}} d t=0 .
\end{gathered}
$$

We can rewrite the first two terms in the numerator as

$$
\sigma_{n}(t) b_{\sigma}^{2}(c)-\sigma(t) b_{\sigma_{n}}^{2}(c)=\sigma_{n}(t) b_{\sigma}^{2}(c)-\sigma(t) b_{\sigma}^{2}(c)+\sigma(t) b_{\sigma}^{2}(c)-\sigma(t) b_{\sigma_{n}}^{2}(c)
$$




$$
\begin{aligned}
& =b_{\sigma}^{2}(c)\left(\sigma_{n}(t)-\sigma(t)\right)+\sigma(t)\left(b_{\sigma}^{2}(c)-b_{\sigma_{n}}^{2}(c)\right) \\
& =b_{\sigma}^{2}(c)\left(\sigma_{n}(t)-\sigma(t)\right)+\sigma(t)\left(b_{\sigma}(c)+b_{\sigma_{n}}(c)\right)\left(b_{\sigma}(c)-b_{\sigma_{n}}(c)\right) .
\end{aligned}
$$

This gives

$$
\begin{gathered}
\int_{C} \frac{\sigma(t)\left(b_{\sigma}(c)+b_{\sigma_{n}}(c)\right)\left(b_{\sigma}(c)-b_{\sigma_{n}}(c)\right)+2 \sigma(t) \sigma_{n}(t)\left(b_{\sigma}(c)-b_{\sigma_{n}}(c)\right)}{\left(b_{\sigma_{n}}(c)+\sigma_{n}(t)\right)^{2}\left(b_{\sigma}(c)+\sigma(t)\right)^{2}} d t \\
+\int_{C} \frac{b_{\sigma}^{2}(c)\left(\sigma_{n}(t)-\sigma(t)\right)+\sigma(t) \sigma_{n}(t)\left(\sigma(t)-\sigma_{n}(t)\right)}{\left(b_{\sigma_{n}}(c)+\sigma_{n}(t)\right)^{2}\left(b_{\sigma}(c)+\sigma(t)\right)^{2}} d t=0
\end{gathered}
$$

Setting $D=\left(b_{\sigma_{n}}(c)+\sigma_{n}(t)\right)^{2}\left(b_{\sigma}(c)+\sigma(t)\right)^{2}$, we have

$$
\begin{aligned}
\left(b_{\sigma}(c)-b_{\sigma_{n}}(c)\right) & \int_{C} \frac{\sigma(t)\left(b_{\sigma}(c)+b_{\sigma_{n}}(c)\right)+2 \sigma(t) \sigma_{n}(t)}{D} d t \\
& =b_{\sigma}^{2}(c) \int_{C} \frac{\sigma(t)-\sigma_{n}(t)}{D} d t+\int_{C} \frac{\sigma(t) \sigma_{n}(t)\left(\sigma_{n}(t)-\sigma(t)\right)}{D} d t
\end{aligned}
$$

Taking the absolute value of both sides and noting that the integral on the left side of the equation is positive, we have

$$
\begin{aligned}
\left|b_{\sigma}(c)-b_{\sigma_{n}}(c)\right| & \int_{C} \frac{\sigma(t)\left(b_{\sigma}(c)+b_{\sigma_{n}}(c)\right)+2 \sigma(t) \sigma_{n}(t)}{D} d t \\
& =\left|b_{\sigma}^{2}(c) \int_{C} \frac{\sigma(t)-\sigma_{n}(t)}{D} d t+\int_{C} \frac{\sigma(t) \sigma_{n}(t)\left(\sigma_{n}(t)-\sigma(t)\right)}{D} d t\right|
\end{aligned}
$$

and so

$$
\begin{aligned}
\left|b_{\sigma}(c)-b_{\sigma_{n}}(c)\right| & \int_{C} \frac{\sigma(t)\left(b_{\sigma}(c)+b_{\sigma_{n}}(c)\right)+2 \sigma(t) \sigma_{n}(t)}{D} d t \\
& \leq b_{\sigma}^{2}(c)\left|\int_{C} \frac{\sigma(t)-\sigma_{n}(t)}{D} d t\right|+\left|\int_{C} \frac{\sigma(t) \sigma_{n}(t)\left(\sigma_{n}(t)-\sigma(t)\right)}{D} d t\right| \\
& \leq b_{\sigma}^{2}(c) \int_{C} \frac{\left|\sigma(t)-\sigma_{n}(t)\right|}{D} d t+\int_{C} \frac{\sigma(t) \sigma_{n}(t)\left|\sigma(t)-\sigma_{n}(t)\right|}{D} d t
\end{aligned}
$$

Now, for a given $\varepsilon$, choose $n$ large enough that $\left|\sigma(t)-\sigma_{n}(t)\right|<\varepsilon$. This 
means

$$
\begin{aligned}
\left|b_{\sigma}(c)-b_{\sigma_{n}}(c)\right| & {\left[\int_{C} \frac{\sigma(t)\left(b_{\sigma}(c)+b_{\sigma_{n}}(c)\right)}{D} d t+2 \int_{C} \frac{\sigma(t) \sigma_{n}(t)}{D} d t\right] } \\
& \leq E^{2} \varepsilon \int_{C} \frac{1}{D} d t+\varepsilon \int_{C} \frac{\sigma(t) \sigma_{n}(t)}{D} d t
\end{aligned}
$$

Dividing both sides by $\int_{C} \frac{\sigma(t) \sigma_{n}(t)}{D} d t$ yields

$$
\begin{aligned}
\left|b_{\sigma}(c)-b_{\sigma_{n}}(c)\right|\left[2+\frac{\int_{C} \frac{\sigma(t)\left(b_{\sigma}(c)+b_{\sigma_{n}}(c)\right)}{D} d t}{\int_{C} \frac{\sigma(t) \sigma_{n}(t)}{D} d t}\right] & \leq \varepsilon\left[1+\frac{E^{2} \int_{C} \frac{1}{D} d t}{\int_{C} \frac{\sigma(t) \sigma_{n}(t)}{D} d t}\right] \\
\left|b_{\sigma}(c)-b_{\sigma_{n}}(c)\right| & \leq \frac{\varepsilon}{2}\left[1+\frac{E^{2} \int_{C} \frac{1}{D} d t}{\int_{C} \frac{\sigma(t) \sigma_{n}(t)}{D} d t}\right]
\end{aligned}
$$

The final step is to establish bounds for $D$. It is clear that $\left(\sigma_{n}(t) \sigma(t)\right)^{2} \leq$ $D \leq\left(E+\sigma_{n}(t)\right)^{2}(E+\sigma(t))^{2}$. Using these bounds, we arrive at

$$
\begin{aligned}
\left|b_{\sigma}(c)-b_{\sigma_{n}}(c)\right| & \leq \frac{\varepsilon}{2}\left[1+\frac{E^{2} \int_{C} \frac{1}{\left(E+\sigma_{n}(t)\right)^{2}(E+\sigma(t))^{2}} d t}{\int_{C} \frac{\sigma(t) \sigma_{n}(t)}{\left(\sigma_{n}(t) \sigma(t)\right)^{2}} d t}\right] \\
& \leq \frac{\varepsilon}{2}\left[1+\frac{E^{2} \int_{C} \frac{1}{\left(E+\sigma_{n}(t)\right)^{2}(E+\sigma(t))^{2}} d t}{\int_{C} \frac{1}{\sigma_{n}(t) \sigma(t)} d t}\right]
\end{aligned}
$$

The right hand side of this inequality does not depend on $c$. This demonstrates that $b_{\sigma_{n}}$ converges uniformly to $b_{\sigma}$. We conclude that the mapping $T$ is continuous. All of the conditions of the Schauder Fixed Point Theorem are satisfied, so $T$ has a fixed point. This proves existence of an equilibrium. 


\section{References}

Bernardo, Antonio, Eric Talley, and Ivo Welch 2000. "A Theory of Legal Presumptions," Journal of Law, Economics, and Organization, 16, 1-49.

Clark, Derek J. and Christian Riis 1998. "Contest success functions: an extension," Economic Theory, 11, 201-204.

Corchón, Luis 2007. "The Theory of Contests: A Survey," Review of Economic Design. forthcoming.

Cornes, Richard and Roger Hartley 2005. "Asymmetric Contests with General Technologies," Economic Theory, 26, 923-946.

Gradstein, Mark 1995. "Intensity of Competition, Entry and Entry Deterrence in Rent Seeking Contests," Economics and Politics, 7, 79-91.

Harstad, Ronald M. 1995. "Privately Informed Seekers of an Uncertain Rent," Public Choice, 83, 81-93.

Higgins, Richard S., William F. Shughart, and Robert D. Tollison 1985. "Free Entry and Efficient Rent Seeking," Public Choice, 46, 247-258.

Hillman, Arye L. and Eliakim Katz 1984. "Risk-Averse Rent Seekers and the Social Cost of Monopoly Power," The Economic Journal, 94, 104-110.

Hillman, Arye L. and John G. Riley 1989. "Politically contestable rents and transfers," Economics and Politics, 1, 17-39.

Hurley, Terrance M. and Jason F. Shogren 1998a. "Asymmetric information contests," European Journal of Political Economy, 14, 645-665.

Hurley, Terrance M. and Jason F. Shogren 1998b. "Effort levels in a Cournot Nash contest with asymmetric information," Journal of Public Economics, 69, 195-210.

Lockard, Alan A. and Gordon Tullock 2001. Efficient Rent-Seeking: Chronicle of an Intellectual Quagmire. New York: Springer-Verlag.

Malueg, David A. and Andrew J. Yates 2004. "Rent Seeking With Private Values," Public Choice, 119, 161-178. 
Malueg, David A. and Andrew J. Yates 2006. "Equilibria in Rent-Seeking Contests with Homogeneous Success Functions," Economic Theory, 27, 719-727.

Nitzan, Shmuel 1991. "Collective Rent Dissipation," The Economic Journal, $101,1522-1534$.

Schoonbeek, Lambert and Barbara M. Winkel 2006. "Activity and Inactivity in a Rent-Seeking Contest with Private Information," Public Choice, 127, $123-132$.

Skaperdas, Stergios 1996. "Contest success functions," Economic Theory, 7, $283-290$.

Szidarovsky, Ferenc and Koji Okuguchi 1997. "On the Existence and Uniqueness of Pure Nash Equilibrium in Rent-Seeking Games," Games and Economic Behavior, 18, 135-140.

Tullock, Gordon 1980. "Efficient Rent Seeking." In Buchanan, James, Robert Tollison, and Gordon Tullock, eds., Toward a Theory of the Rent-Seeking Society, pages 97-112. College Station, TX: Texas A \& M Univ. Press.

Van Long, Ngo and Neil Vousden 1987. "Risk-Averse Rent Seeking with Shared Rents," The Economic Journal, 97, 971-985.

Wärneryd, Karl 2003. "Information in Conflicts," Journal of Economic Theory, $110,121-136$.

Wärneryd, Karl 2006. "Participation in Contests with Asymmetric Information," Stockholm School of Economics.

Zeidler, Eberhard 1986. Nonlinear functional analysis and its applications. I Fixed-point theorems. New York: Springer-Verlag. Translated from the German by Peter R. Wadsack. 